\title{
Bi-Tm (Bismuth-Thulium)
}

\section{H. Okamoto}

[1999Oka] showed the Bi-Tm phase diagram reported by [1996Abd] and pointed out that the shape of the BiTm liquidus must be reexamined because its extension to the 0 at.\% Tm side would cross the pure Bi line unless a very unlikely abrupt change of slope is introduced. This thermodynamically unlikely situation was "confirmed" by [2003Abu] by means of DTA, x-ray diffraction, and microstructural analysis, as reported by [20060ka].

However, [20060ka] still strongly suspected the shape of the Bi-Tm liquidus near the Bi-rich end of the phase diagram.

Figure 1 shows the Bi-Tm phase diagram thermodynamically assessed by [2010Wan]. The phase boundary data used in this assessment were primarily based on [2003 Abu]. The appearance of the BiTm liquidus near the Bi-rich end is normal in Fig. 1. Accordingly, this phase diagram is preferred to the one shown in [20060ka].

\section{References}

1996Abd: M.N. Abdusalyamova, A.G. Chuiko, E.I. Shishkin, and O.I. Rachmatov, Phase Diagrams and Thermodynamic Properties of Rare Earth-Bismuth Systems, J. Alloys. Compd., 1996, 240, p 272-277

19990ka: H. Okamoto, Bi-Tm (Bismuth-Thulium), J. Phase Equilib., 1999, 20(2), p 164

2003Abu: V.D. Abulkhaev, Phase Relations and Properties of Alloys in the Tm-Bi System, Neorg. Mater, 2003, 39(1), p 54-57, in Russian, TR: Inorg. Mater, 2003, 39(1), p 4749

2006Oka: H. Okamoto, Bi-Tm (Bismuth-Thulium), J. Phase Equilib., 2006, 27(3), p 305

2010Wan: C.P. Wang, H.L. Zhang, A.T. Tang, F.S. Pan, and X.J. Liu, Thermodynamic Assessments of the Bi-Nd and Bi-Tm Systems, J. Alloys Compd., 2010, 502, p 43-48

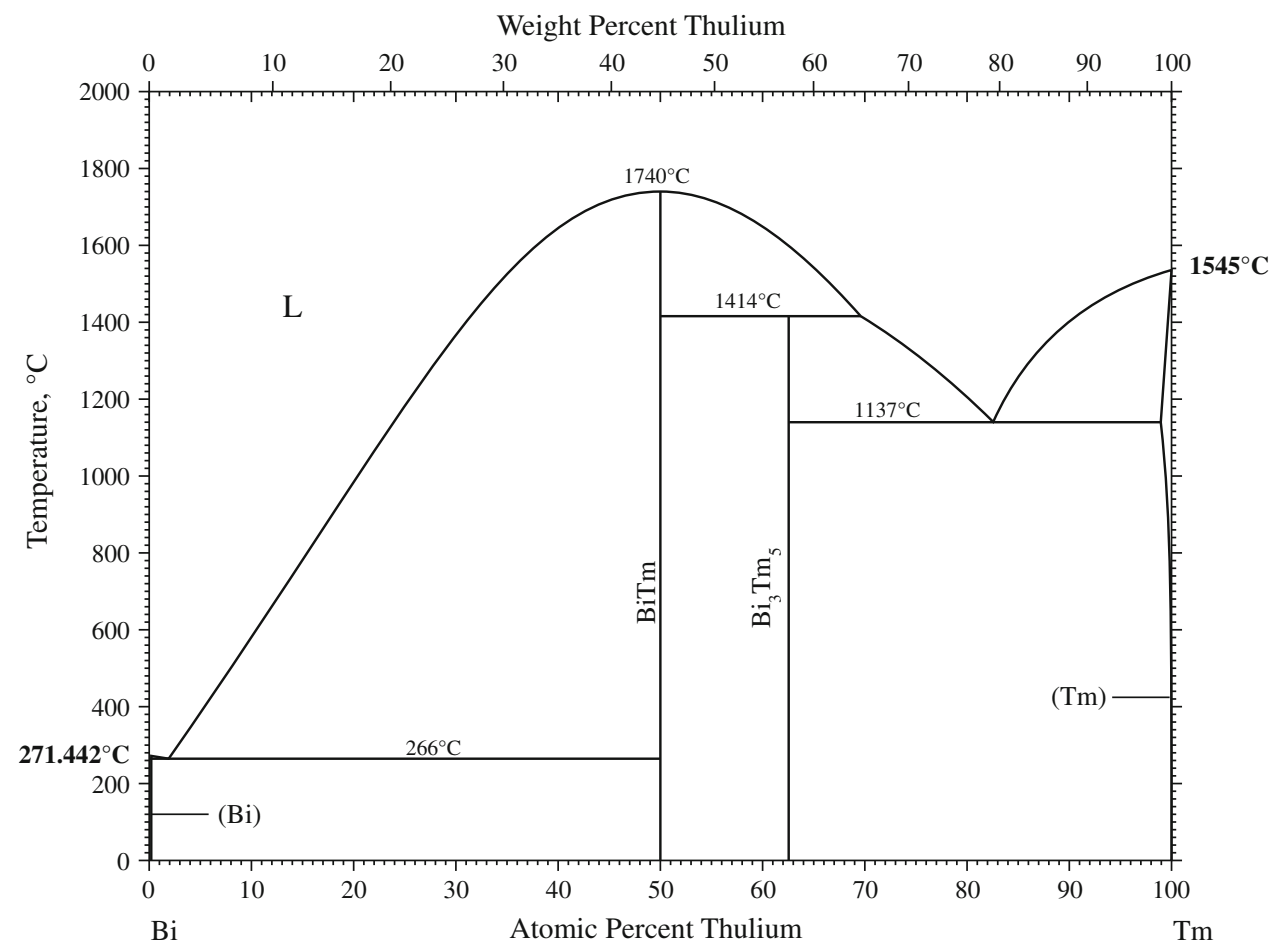

Fig. 1 Bi-Tm phase diagram 